\title{
Essais
}

ESSAIS

Revue interdisciplinaire d'Humanités

Hors-série 4 | 2018

Stanley Kubrick

\section{Paths of Glory. Aux croisements de l'Histoire}

\section{Clément Puget}

\section{(2) OpenEdition}

Journals

Édition électronique

URL : http://journals.openedition.org/essais/574

DOI : 10.4000/essais. 574

ISSN : 2276-0970

\section{Éditeur}

École doctorale Montaigne Humanités

\section{Édition imprimée}

Date de publication : 1 juillet 2018

Pagination : 43-57

ISBN : 979-10-97024-04-8

ISSN : 2417-4211

Référence électronique

Clément Puget, «Paths of Glory. Aux croisements de l'Histoire », Essais [En ligne], Hors-série 4 | 2018, mis en ligne le 01 décembre 2019, consulté le 16 décembre 2019. URL : http://

journals.openedition.org/essais/574 ; DOI : 10.4000/essais.574 


\section{Paths of Glory. Aux croisements de l'Histoire}

\section{Clément Puget}

Si Les Sentiers de la gloire est évidemment un film célèbre - adjectif qui pourrait qualifier les 13 films de Stanley Kubrick... - ce qui distingue peut-être celui-ci des autres, qui le précèdent ou le suivent, tient d'abord au contexte présent. Il n'est pas inexact de dire que l'on commémore, en 2017, le $60^{\mathrm{e}}$ anniversaire du film sorti aux États-Unis en décembre sous le titre de Paths of Glory. C'est un film de guerre, écrivent alors les critiques, où plutôt un film dans lequel guerres et conflits trouvent un ancrage historique, et ce à l'instar de la moitié des films du cinéaste finalement. Fear and Desire, œuvre inaugurale éternellement mal-aimée du maître - film de "n'importe quelle guerre en somme " aux « contours immuables de la peur, du doute et de la mort " ouvrit les hostilités avant que Spartacus ne permette à Kirk Douglas d'abandonner l'uniforme français du colonel Dax tout en demeurant plein-cadre. Hormis ces deux films - les moins "kubrickiens" ? - suivent Dr. Strangelove, Barry Lyndon ou encore Full Metal Jacket, soit autant d'évocations poétiques et historiennes à la fois de conflits modernes et contemporains, belliqueux et/ou diplomatiques...

Mais si Paths of Glory m'intéresse avant tout, sans doute est-ce en raison de cette longue lutte dans laquelle je me suis engagé pour essayer encore et toujours d'imaginer un pont entre deux rives de la recherche universitaire longtemps demeurées distantes, qui pourtant se font face tels des duellistes, depuis la fin du XIX siècle : l'Histoire d'un côté, le cinéma de l'autre. Comme tout chercheur ayant abordé les représentations de la Grande Guerre au cinéma, j’avais croisé le quatrième long métrage de Stanley Kubrick pendant la rédaction de ma thèse de doctorat, publiée sous le titre Verdun, le cinéma, l'événement ${ }^{2}$. Je concluais d'ailleurs cet ouvrage relatif à l'écriture de la bataille de Verdun en interrogeant l'année 1916 comme point d'ancrage notoire dans la mémoire collective française, année médiane du conflit mais égale-

Stanley Kubrick, Fear and Desire (1953), traduction réalisée par nos soins.

2 Puget Clément, Verdun, le cinéma, l'événement, Paris, Nouveau monde éditions/Ministère de la Défense, collection " Histoire et cinéma ", 2016, 543 p. 
ment année des batailles monumentales de la Somme et bien évidemment de Verdun. Étrange hasard - passée la Marseillaise orchestrée du générique de début - Paths of Glory s'ouvre sur une surimpression filmique : "France $1916 »$ (Document 1).

Ce film, qui fit connaître Kubrick à l'international - après les succès relatifs de ses films de genre, Killer's Kiss et The Killing - est d'autant plus illustre qu'il semble en avoir engendré d'autres, tels les non moins importants King and Country (Joseph Losey, 1964), Uomini contro (Francesco Rosi, 1970) ou Capitaine Conan (Bertrand Tavernier, 1996), sans oublier les productions télévisuelles d'Yves Boisset ou Patrick Jamin notamment. Et si Westfront 1918 ("Quatre de l'infanterie ", G.W. Pabst) ou All Quiet on the Western front égratignaient déjà, à l'orée des années 1930, l'absurdité des ordres hiérarchiques et le bellicisme des nations, Paths of Glory choisit quelques pans précis de la Grande Guerre, dans ce qu'elle recèle d'abjection, de cruauté et d'injustice inouïe. Donc l'analyser en observant les nouveaux horizons de la recherche kubrickienne est un défi car Paths of Glory suggère avant tout les débuts - les origines peut-être même de la carrière mondiale d'un metteur en scène et la confirmation d'une éclosion qui fit surtout l'effet d'une explosion analogue à celle de l'artillerie lourde qui habite le hors-champ de Paths of Glory.

Pourquoi Kubrick s'est-il plongé dans l'enfer des tranchées mais aussi dans l'ambiance glaçante des lieux de pouvoir d'une guerre lointaine (1914-1918) qui en appelaient certainement d'autres en 1957 ? Peut-être, alors que la guerre d'Algérie se répandait à l'ensemble du territoire français et que les États-Unis peinaient à faire oublier la guerre de Corée, Kubrick eut-il l'idée de réaliser un film - comme aurait pu le dire Abel Gance ou Henri Desfontaines - pour "tuer la guerre"!

Après Fear and Desire (1953) qui avait quelque chose d'une œuvre " théorique ", puis deux films noirs - Killer's kiss et The Killing - Stanley Kubrick adapte donc un ouvrage de l'écrivain américain et ancien-combattant, Humphrey Cobb, engagé dans l'armée canadienne en 1916 pour combattre en France, où il sera gazé mais reviendra de la guerre ${ }^{3}$. Cobb écrit ce roman fondateur en 1935 et meurt en 1944, sans jamais avoir eu vent du projet de Stanley Kubrick d'adapter Paths of Glory, vingt deux ans après la sortie de l'ouvrage. Du roman, d'ailleurs adapté au théâtre par Sidney Howard en 1935, Kubrick et ses coscénaristes, Willingham et Thompson, ont gardé la structure tripartite éliminant cependant quelques protagonistes, faisant d'Étienne et Dax, par exemple, un seul et même officier, défenseur, combattant et avocat de ses hommes, dans l'adversité insoupçonnée de l'État-major français. Mais plus que tout autre motif ou parole puisée dans l'ouvrage, Kubrick est resté fidèle à l'ironie originelle et fondatrice du roman, ainsi que le démontre la séquence d'exécution des trois soldats. Nous y reviendrons.

3 Cobb Humphrey, Les Sentiers de la gloire, éd. Altal, Chambéry 2014, 269 p. 
Croisant donc « Histoire » et " cinéma » tant dans mes enseignements qu'au sein de mes recherches (actuelles également sur la commémoration médiatique et audiovisuelle de l'événement historique), il me semble possible d'interroger ce film selon trois axes qui pourraient répondre à la question suivante : dans quelle mesure le film Paths of Glory construit-il son historicité (Rancière ${ }^{4}$ ) au croisement de l'Histoire et de l'histoire, débordant et reconfigurant le cadre chronologique de la Grande Guerre tout en développant le thème du "refus de guerre " dans différentes strates de son récit, et cela avec quelles conséquences sur la réception de l'œuvre des années 1960 aux années 1990 ?

\section{L'historicité du film et les arrangements avec l'Histoire}

\section{Un lieu dans l'histoire}

The anthill, la " fourmillière » empruntée au roman de Cobb est un point stratégique manifeste dans les deux récits, écrit de 1934 et filmique de 1957. Mirage (d'ailleurs presque invisible à l'œil nu du spectateur), cet ouvrage fortifié pris, perdu et repris par l'ennemi, semble passer de main en main au gré du pilonnage des canons (de 420 ?) et des vagues d'assaut de l'infanterie. Si aucun lieu n'est clairement mentionné, ni dans le roman, ni dans le film, on peut s'interroger sur les motifs visuels dans Paths of Glory d'une part et à travers deux photogrammes de Verdun visions d'Histoire (L. Poirier, 1928), tourné à Verdun et notamment au fort de Douaumont, d'autre part (Document 2).

En 1916, ainsi que le relatait déjà Léon Poirier dans son film de 1928, le fort de Douaumont fait l'objet de plusieurs prises et pertes par les Français avant d'être repris définitivement le 24 octobre 1916 - une semaine avant un autre fort illustre, celui de Vaux.

Stanley Kubrick y fait-il indirectement référence ? Peut-être a-t-il d'ailleurs été inconsciemment influencé par le roman qui mentionne une fois, mais une seule, le nom de Verdun (p. 195) dans un moment de flash-back du récit ? S'il est impossible de l'affirmer, l'ancrage du récit de 1957 dans l'armée française couplé à la surimpression " France 1916 " peut laisser penser qu'un tel rapprochement n'est peut-être pas anodin.

\section{Historio-grahie et «mémoire de seconde main»}

Le deuxième point notable est relatif à l'attaque menée par le colonel Dax dans le no man's land à l'assaut de la fourmilière.

4 Rancière Jacques, "L'historicité du cinéma » in Antoine de Baecque, Christian Delage (éd.), De l'histoire au cinéma, éd. Complexes IHTP, Bruxelles 1998, p. 45-60. 
Cette attaque suicide est collectivement refusée par une partie de la compagnie qui décide de ne pas sortir de la tranchée. Kubrick a repris l'intégralité de l'épisode développé dans le roman faisant de l'ordre du général Assolant - «Mireau » dans le film - le point d'orgue de cette séquence (Document 3). Terrifiant, ce moment trouve difficilement son fondement dans une chronologie précise. Cobb s'est certainement inspiré de l'affaire dite de Souain, pour interpréter les rumeurs d'un tel ordre historiquement avéré et donné, en mars 1915, par le général Reveilhac ${ }^{5}$. En effet, Hormis les doutes exprimés par plusieurs historiens au sujet de la véracité d'une telle décision, si celle-ci eut cependant lieu, ce fut en 1915, soit environ un an avant les faits rapportés dans le film de Stanley Kubrick.

Si l'on envisage l'historicité de l'œuvre, au-delà du simple rapport entre le fait réel attesté par les archives et le fait strictement filmique, et que l'on regarde du côté du film de Georg Wilhelm Pabst, on découvre que l'historicité recherchée par Stanley Kubrick réside peut-être moins dans un quelconque champ de bataille ou date précise que... dans un autre film, Westfront 1918 dans lequel une scène analogue - côté allemand cette fois - est scénarisée et mise en images. Une manière de dire peut-être que l'historicité est ici surtout le rapport de l'événement filmique à l'événement médiatisé, une " mémoire de seconde main » en somme.

\section{Le «Shell-shock» (Document 4)}

Cette expression britannique date de 1915. Elle désigne les atteintes d'ordre nerveux et psychique provoquées sur l'organisme humain par les déflagrations d'explosifs. Le terme a progressivement été banni du vocabulaire médical militaire en raison de l'usage néfaste qui pouvait en être fait au sein des troupes. La langue française offre plusieur équivalents outre "l'état de choc » tel que traduit dans le film : commotion cérébrale, congestion cérébrale, accidents nerveux, commotions médulaires, choc émotionnel, obusite se répandent après-guerre ${ }^{6}$. Cependant deux psychiatres français, Dumas et Delmas, font un rapport pendant la bataille de la Somme (octobre 1916), sur la notion de syndrome confusionnel, pour alerter les autorités militaires des séquelles tenaces dues aux épisodes de confusion mentale, et insister sur les conséquences tragiques de ces séquelles sur le plan médico-légal. Ils y indiquent que les " non-soignés " car " non-diagnostiqués " comme tels conservent une hyperémotivité durable qui se traduit sur le champ de bataille par des

5 Offenstadt Nicolas, Les Fusillés de la Grande Guerre et la mémoire collective 1914-1999, éd. Odile Jacob, Paris, 2002 [1999].

6 Dupouy Stéphanie, "La vérité troublée. Georges Dumas, psychiatre du front ", in Christophe Prochasson, Anne Rasmussen (éd.), Vrai et faux dans la Grande Guerre, éd. La Découverte, Paris, 2004, p. 235-254. 
"phobies de l'obus » ou des réactions de fuite pathologique, les conduisant, les cas échéant à déserter :

"Ces hommes sont pour longtemps encore des infirmes du système nerveux; ce n'est pas quelques années de prison ou le poteau d'exécution que nous leur devons mais des soins et du repos dont nul ne songerait à contester l'utilité si leur infirmité provenait d'une blessure apparente. " ${ }^{7}$

Loin de conter une bataille, encore moins une année de luttes plus ou moins intenses - comme la surimpression "France 1916 » aurait pu inciter à le penser - Paths of Glory prend surtout le chemin d'une représentation éclatée, parcellaire, de l'Histoire avec un récit dont la volonté est de synthétiser les maux de la guerre... en 1916. Ce tableau général du conflit, esquissé en amont, Kubrick l'a voulu plus précis encore et contextualisé sur le cas de l'exercice de la justice (militaire) et des exécutions, toujours en étroite corrélation avec le contenu de l'ouvrage de l'ancien-combattant Humphrey Cobb.

\section{Justice militaire}

Trois affaires de fusillés et leur exécution filmique

Kubrick, fidèle à l'ouvrage de Humphrey Cobb, a repris les trois affaires dites de fusillés de la Grande Guerre, pour bâtir son récit. Ce sont d'abord des lieux : Roye, dans la Somme (septembre 1914), Souain (mars 1915), Flirey (avril 1915). Aucune donc ne se déroulât en 1916...

De la plus ancienne affaire (septembre-octobre 1914), le film retient le cas du sous-lieutenant Chapelant, officier donc qui, du côté de la Somme aux environs de Roye, s'est rendu à l'ennemi avec sa troupe pensant que les troupes allemandes encerclaient leurs positions. Blessé (par une balle française), le sous-lieutenant Chapelant est ramené dans les lignes françaises sur un brancard... et condamné à mort. Ne pouvant se tenir debout, il est fusillé sur ce même brancard face au Château des loges, près de Roye (Document 5 ).

Ce n'est pas son histoire qui est contée à l'écran mais une évocation certaine de cette victime réhabilitée par l'État français en 2012 au moment des commémorations nationales du 11 novembre. Car comme l'écrivit Alain, dès 1927, on ne savait rien de précis de cette affaire, que l'atroce exécution...

Dans la chronologie de la Guerre, l'épisode de Souain (mars 1915) fournit matière au récit de $\mathrm{H}$. Cobb, avec le choix de quatre caporaux, les plus jeunes du régiment, dont le caporal Maupas, fusillés pour l'exemple face à la multitude qui a refusé de combattre (au départ, pas moins de vingt-quatre hommes étaient menacés d'exécution) (Document 6).

7 Propos de Georges Dumas, in Stéphanie Dupouy, ibid., p. 247. 
Enfin, de Flirey en avril 1915, Stanley Kubrick retient surtout le personnage du capitaine (ici le colonel Dax interprété par Kirk Douglas) qui fut chargé de la défense - seulement quelques heures avant le procès - de cinq hommes condamnés pour avoir refusé d'aller au combat.

De ces trois affaires émergent quatre hommes, dans le roman de Cobb, tous soldats de troupe. Mais le quatrième, parce qu'il est juif, va être épargné par le tirage au sort des victimes - procédé qui ne se pratiquait pourtant plus dans l'armée française à partir du printemps 1916.

Dans le film éponyme, Stanley Kubrick a retiré le quatrième personnage de son intrigue, faisant des fusillés, des victimes de la barbarie du commandement français. Il représente l'instant décisif de manière très précise, suivant le décret de 1909, avec un peloton constitué de tireurs appartenant à la même unité que les condamnés. La parole de l'institution militaire et celle du condamné sont les seules paroles publiques audibles durant la cérémonie. Mais si cet acte final d'une tragédie annoncée se conforme avec précision aux récits historiens sur le sujet, ce climax est précédé par un simulacre d'exercice de justice militaire qui, conformément au ton général du récit filmique, mêle une fois encore anachronisme et réalité sensible de la Grande Guerre dans un alliage d'historicité aussi étrange que séduisant. Pertinent peut-être aussi...

\section{L'in-justice militaire}

Le décor du château de Schleissheim en Bavière fournit le cadre d'un procès aux accents baroques mais également caricaturaux de bout en bout de la séquence filmique.

Tourné en Allemagne, au dit château et également dans les studios munichois de Geseilgasteig, pour des raisons budgétaires semble-t-il, avec des figurants essentiellement allemands issus de la police locale, cette séquence dévoile le procès et la tenue d'une cour martiale pour juger les trois soldats (Document 7).

Devant ce que l'on serait tenté de qualifier de caricature, on peut se demander comment la justice militaire s'applique pendant la Première Guerre mondiale ? Il s'agit d'une justice militaire d'exception en 1914, se fondant sur le code de justice militaire en vigueur depuis 1857 . Le 2 août 1914, un décret annonçait l'état de siège, le territoire devenant - jusqu'en 1919 - «zone des armées ", en vertu de quoi la justice qui s'y appliquait était une justice militaire, selon l'application de la loi du 9 août 1849 sur l'état de siège. Et si, avant 1914, la peine de mort est très peu pratiquée et appliquée dans le cadre de la justice militaire, la situation en 1914 et la patrie est en danger, transforment l'appareil judiciaire en une justice de terreur. Dès la fin août 1914, les exécutions sommaires sont autorisées. 
À partir du 27 avril 1916, les choses changent cependant, lorsque le parlement vote une loi visant à restreindre les pouvoirs spéciaux des cours de justice militaire, cela pour que la "saignée " pratiquée en 1914-1915 cesse enfin, notamment via l'abolition du tirage au sort en cas de condamnation collective. Notons à cet égard que seule une minorité d'hommes fut passée par les armes, dans l'armée française, entre 1914 et 1918, soit tout de même plus de 700 sur environ 2500 condamnations à mort. André Loez rapporte que la majorité des fusillés a été exécutée entre 1914 et 1915 , soit en début de guerre donc pour un total de 500 fusillés en un an et demi, alors que les années 1916-1918 affichent un bilan de 250 hommes exécutés - soit la moitié ${ }^{8}$. Curieusement, du moins à l'inverse de ce que l'imaginaire national français a retenu - et les manuels scolaires dans une certaine mesure -, il y eut donc moins de soldats passés par les armes après 1916. Par conséquent, la violence de l'armée et sa fermeté ne sont pas liés à une guerre qui s'éternise, ce qui renforce la pertinence et l'historicité du choix de situer le film Paths of Glory en 1916 (Document 8).

Nous l'avons dit, Kubrick choisit d'ancrer sa diégèse en 1916, ce qui est intéressant par rapport à l'historiographie sur le sujet, présentant généralement l'imaginaire de 1916 au regard de celui de 1917 (année des mutineries de l'armée française). C'est pourtant aussi en avril 1916 que les conseils de guerre spéciaux sont supprimés, suite à la pression médiatique et populaire et à l'enlisement du conflit qui ne mobilise guère plus les esprits... Vu les remarques de Dax, outré par le caractère expéditif du procès on comprend pourtant que la procédure n'est pas respectée. Pour le spectateur, se posait alors la question : le "conseil de guerre " ici ne ressemble-t-il pas plus aux tribunaux militaires de la guerre d'Algérie dont les pouvoirs spéciaux sont accrus dès 1956, après que l'état d'urgence a été décrété en 1955, qu'à ceux de $1916 \ldots$ ? En Algérie encore, les procédures de justice simplifiée permettent la traduction, sans instruction, des flagrants délits commis avec armes ou matériel militaire en Algérie - justice pour le moins expéditive qui a peut-être inspiré Stanley Kubrick... et lui a causé quelques difficultés diplomatiques aussi au moment de la sortie du film.

\section{« Eyes wide shut ». Réception et aveuglement diplomatique dans la France des années 60 à 90}

Sorti à la veille des fêtes de fin d'année du côté de New York en 1957, le film ne trouva d'écran en France qu'en mars... 1975. Pourquoi ? Laurent Véray a bien exposé les raisons de cette "non-sortie " en France en 1958, suite aux

8 Loez André, 14-18. Les refus de la guerre. Une histoire des mutins, éd. Gallimard, collection Folio Histoire, Paris, 2010, 690 p. 
troubles occasionnés en Belgique, qui auraient incité le distributeur américain United Artists à renoncer à présenter le film à la censure? . En réalité, deux mois avant que le film ne soit projeté en Belgique en janvier 1958, Romain Gary, écrivain et consul général de France à Los Angeles s'opposât à la sortie du film dans l'Hexagone, via une lettre adressée à l'ambassadeur de France à Washington. Il y dénonçait l'antimilitarisme de l'œuvre et le ton calomnieux proféré à l'égard de l'armée française et de son État-major. L'inquiétude du diplomate visait surtout le statut de la France en tant que puissance militaire dans le monde. En effet, en marge de la défaite de Dien Bien Phu (mai 1954), la reconquête coloniale s'amorçait en Algérie. Cependant en 1957, l'échec de la bataille d'Alger et les révélations sur les exactions commises par l'Armée - comparées dans Le Monde à celles perpétrées par les Nazis en leur temps assènent un coup médiatique terrible à la France.

Ainsi comprend-on aisément pourquoi Romain Gary s'inquiète de la portée d'un tel film alors que le pouvoir militaire français est pris d'une terrible paranoïa qui le gangrène irrémédiablement. Lorsque la Belgique informe la France de ce qui s'est passé lors des séances de projection, le ministre des Affaires étrangères indique à l'ambassadeur de France aux États-Unis de tout mettre en œuvre pour empêcher que le film ne soit projeté. Et la France de menacer United Artists de bloquer l'entrée de ses films pendant trois mois. La firme américaine accepte de ne pas présenter le film à la Censure, ce qui permet à l'État français d'indiquer qu'il n'a jamais interdit le film...

S'en suivent quelques interdictions, bien réelles celles-là, dans plusieurs pays, grâce à la pression exercée par les ambassadeurs français en Suisse et Israël par exemple. Au Canada, l'interdiction est plus difficile à réaliser, les étudiants manifestant leur mécontentement dans la lignée de leurs prises de position contre la politique coloniale française en Algérie. À Berlin, les projections sont interrompues en début d'année 1958 pour reprendre en juillet.

Il y a beaucoup d'ironie dans cet épisode de fausse censure et de vraie pression politique et diplomatique à l'encontre de l'art. Ironie et noirceur kubrickiennes sont donc de mise - repensons simplement au soldat blessé suite à une bagarre dans sa cellule, qui lui vaudra de mourir sur le brancard dans une scène qui confine au ridicule et pourtant à l'historicité manifeste... -sans verser dans la gratuité d'un propos loufoque. Non, l'ironie et la noirceur semblent donc être les maitre-mots de ce quatrième long métrage de Stanley Kubrick, qui aborde ce que l'historien George Mosse appellera, dès les années 1980, "the brutalisation" des sociétés en guerre ${ }^{10}$.

9 Véray Laurent, "Le cinéma américain constitue-t-il une menace pour l'identité nationale française ? le cas exemplaire des Sentiers de la gloire (Paths of Glory, 1957, Kubrick) ", in Martin Barnier, Raphaëlle Moine (éd.), France / Hollywood, L'Harmattan, Paris, 2002.

10 Mosse George, De la Grande Guerre au totalitarisme. La brutalisation des sociétés européennes, éd. Hachette Littératures, Paris, 1999, 291 p. 
Paths of Glory n'est donc pas un énième récit plus ou moins fidèle relatant des événements de la Grande Guerre. C'est peut-être d'abord un film qui déjà se retourne sur la jeune carrière de son auteur, faisant écho au tout début de son premier film de long-métrage, Fear and Desire (1953), dont voici la retranscription/traduction du commentaire off des plans inauguraux :

"[...] There is war in this forest. Not a war that has been fought, or one that will be. But any war, and the ennemies who struggle here do not exist, unless we call them into being. This forest then, and all that happens now is outside of History. Only the unchanging shapes of fear and doubt, and death, are from our world. ${ }^{11}$

\title{
Clément Puget
}

Maître de conférences Université Bordeaux Montaigne Unité de recherche CLARE (EA 4593) - équipe Artes Chercheur associé IRCAV Paris 3 clement.puget@u-bordeaux-montaigne.fr

\begin{abstract}
Résumé
Paths of Glory, adaptation filmique du roman éponyme de Humphrey Cobb, dont les 60 ans sont célébrés en 2017, n'est pas un énième film relatant la Grande Guerre. Non, c'est d'abord une œuvre qui déjà se retourne sur la jeune carrière de son auteur, en 1957, faisant écho au tout début de son premier film de long-métrage, Fear and Desire (1953). Mais au-delà de cet évident rapport, c'est celui du film aux événements historiques et à leur historiographie qui fait tout l'intérêt du $4^{\mathrm{e}}$ opus de Stanley Kubrick dans lequel humanisme, précision historienne et ironie macabre se croisent à la fois " hors de l'histoire " (si l'on pense aux anachronismes et invraisemblances du récit) mais également tout en poursuivant l'esquisse du simulacre de guerre dont les « contours immuables de la peur, du doute et de la mort » sont les plus évidents stigmates d'un réel passé qui interroge le cinéaste.
\end{abstract}

\section{Mots-clés}

Fusillé, Grande Guerre, refus, combat, justice, historicité.

11 «La guerre est présente dans cette forêt. Ni pourtant une guerre réelle, ni une guerre qui le deviendra. N'importe quelle guerre en somme, dans laquelle les ennemis qui s'opposent ici n'existent pas, à moins que nous ne les convoquions dans notre réalité. Cette forêt donc, et tout ce qui s'y joue est hors de l'Histoire. Seuls les contours immuables de la peur, du doute et de la mort appartiennent à notre monde. » (traduction réalisée par nos soins). Stanley Kubrick, Fear and Desire (1953). 


\section{Abstract}

Paths of Glory, the film adaptation of Humphrey Cobb's eponymous novel-whose $60^{\text {th }}$ anniversary was celebrated in 2017- is not yet another film relating the Great War. This is first and foremost a work which, in 1957, already looks back on its author's short career, by echoing Kubrick's first feature film, Fear and Desire (1953). But beyond this obvious connection, the film's connexion to historical events and to their historiographies is of central importance in Stanley Kubrick's $4^{\text {th }}$ work; a film in which humanism, historical precision and macabre irony intermingle "out of history" -if one thinks of the narrative's anachronisms and improbabilities - while simultaneously pursuing the depiction of a simulacrum of warfare whose "immutable contours of fear, doubt and death" are the most obvious stigmata of a real past which the filmmaker questions.

\section{Keywords}

Firing squad, Great War, disobedience, battle, justice, historicity. 


\section{NOTICE BIOGRAPHIQUE}

Clément Puget, historien de formation, maître de conférences en Cinéma et audiovisuel à l'Université Bordeaux Montaigne, est qualifié en $18^{\mathrm{e}}$ et $22^{\mathrm{e}}$ sections du CNU. Enseignantchercheur, il est membre de l'unité de recherche CLARE et du centre Artes (Université Bordeaux Montaigne) ainsi que chercheur associé à l'IRCAV (Paris 3 Sorbonne nouvelle). Il est spécialiste des rapports entre Histoire et cinéma. Ses travaux relatifs aux archives, à la mémoire et à l'écriture de l'Histoire, au cinéma et à la télévision, portent sur la période de la Grande Guerre, et notamment sur le cas de la bataille de Verdun dans les films d'actualité, documentaires et de fiction, de long et court métrage. Il est l'auteur de Verdun, le cinéma, l'événement (Nouveau monde éditions, 2016). Dans le cadre du programme de recherches ANR Ciné 08-19 dont il est membre du consortium, il codirige le séminaire de recherches impliquant plusieurs universitaires et représentants de centres d'archives, en partenariat avec la Mission du centenaire 14-18. Coresponsable, depuis 2012, du Master Cinéma et audiovisuel parcours "Documentaire et archives ", de l'Université Bordeaux Montaigne, ses recherches actuelles portent sur la commémoration audiovisuelle de l'événement historique dans les programmes de télévision français. Il coordonne actuellement la rédaction d'un ouvrage collectif relatif à l'œuvre audiovisuelle de l'historien Marc Ferro. 


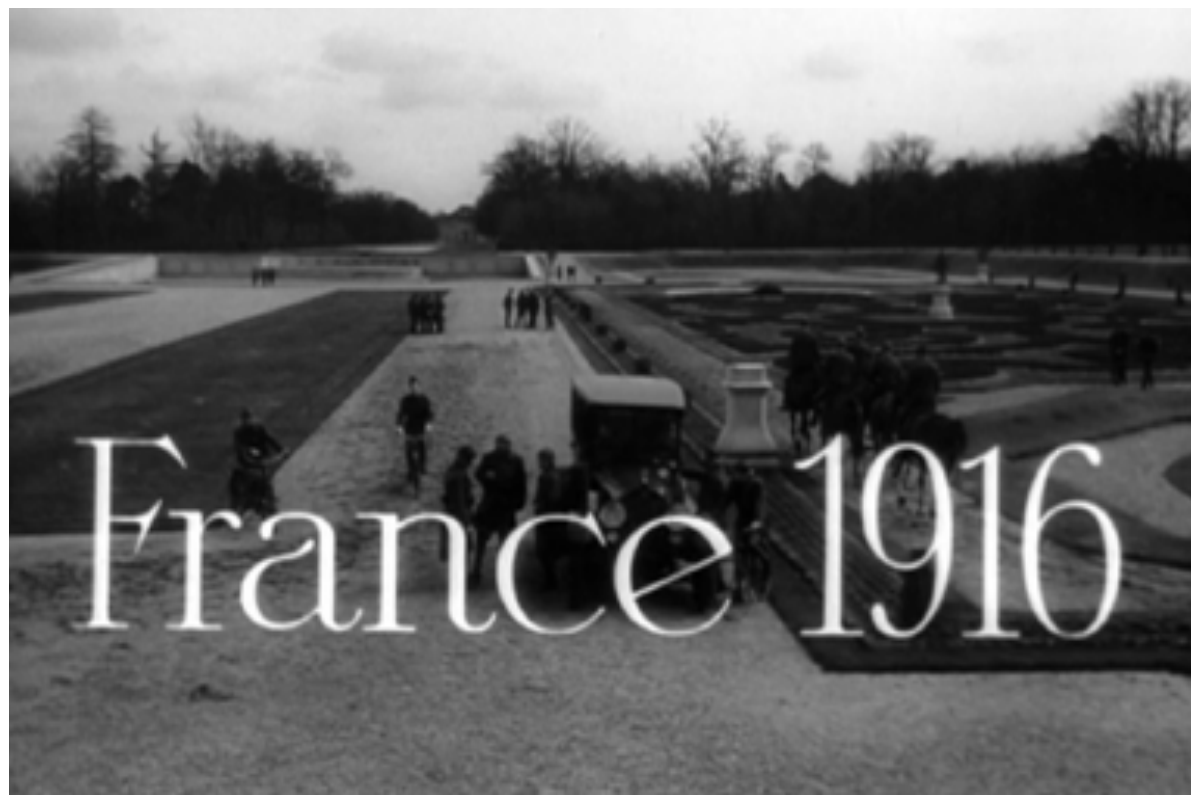

Document 1 : Surimpression initiale, post-générique.

Paths of Glory (Stanley Kubrick, 1957, Bryna productions, United Artists), DR.

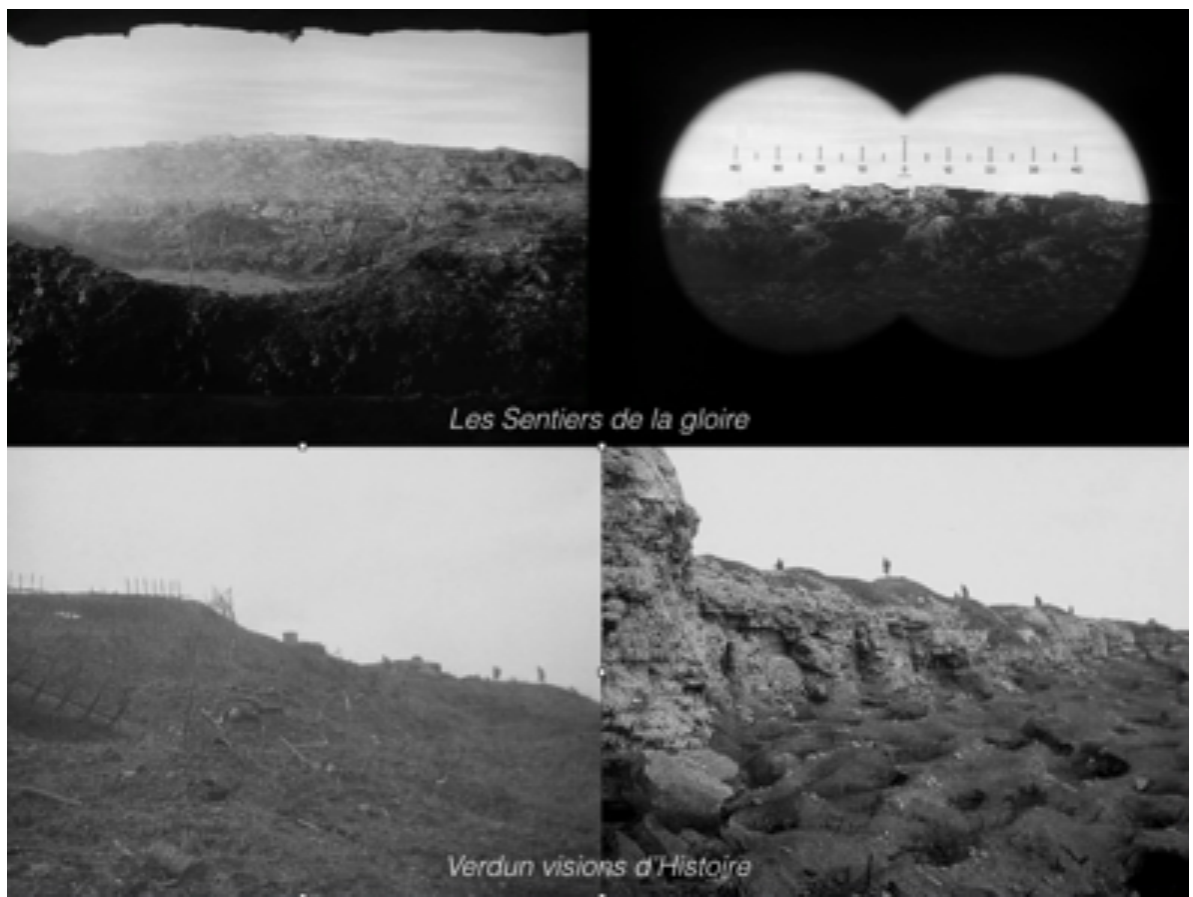

Document 2 : La fourmillère/Douaumont

Paths of Glory (Stanley Kubrick, 1957, Bryna productions, United Artists), DR. Verdun, visions d'Histoire (Léon Poirier, 1928, La cinémathèque de Toulouse), DR. 


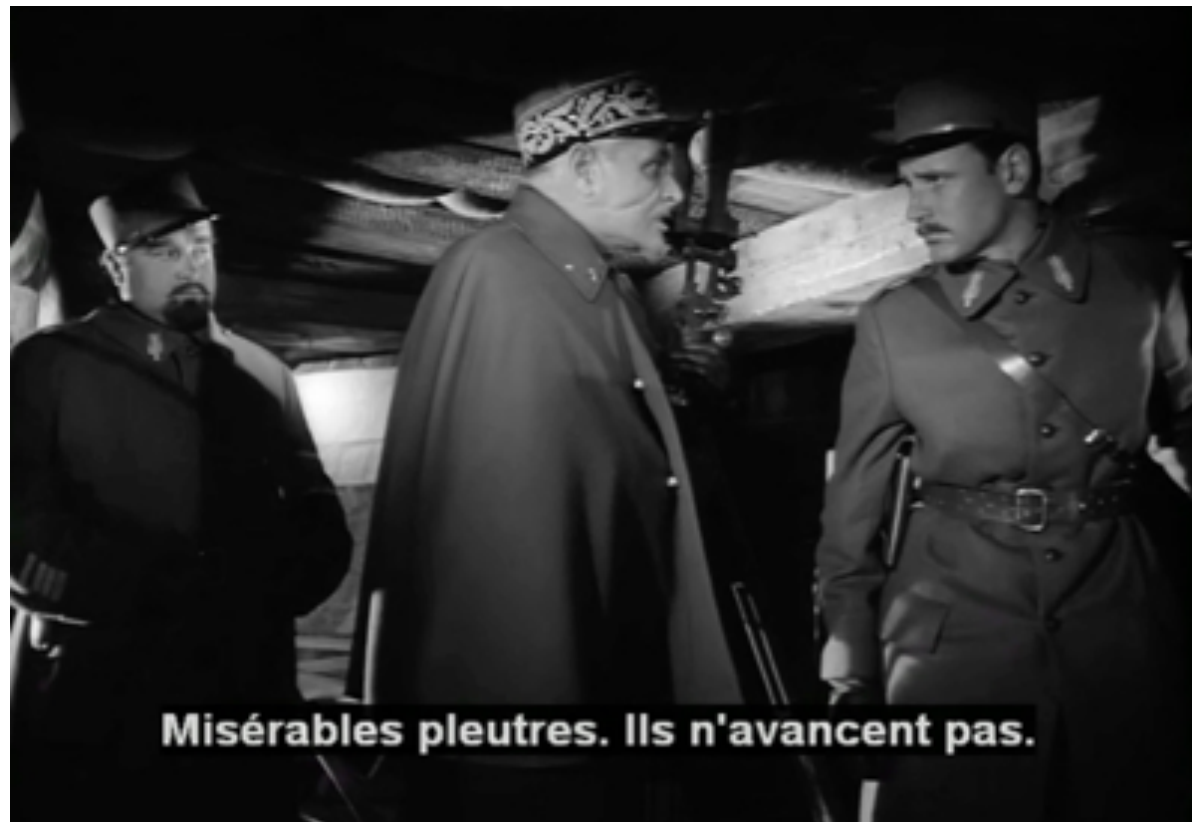

Document 3 : General Mireau

Paths of Glory (Stanley Kubrick, 1957, Bryna productions, United Artists), DR.

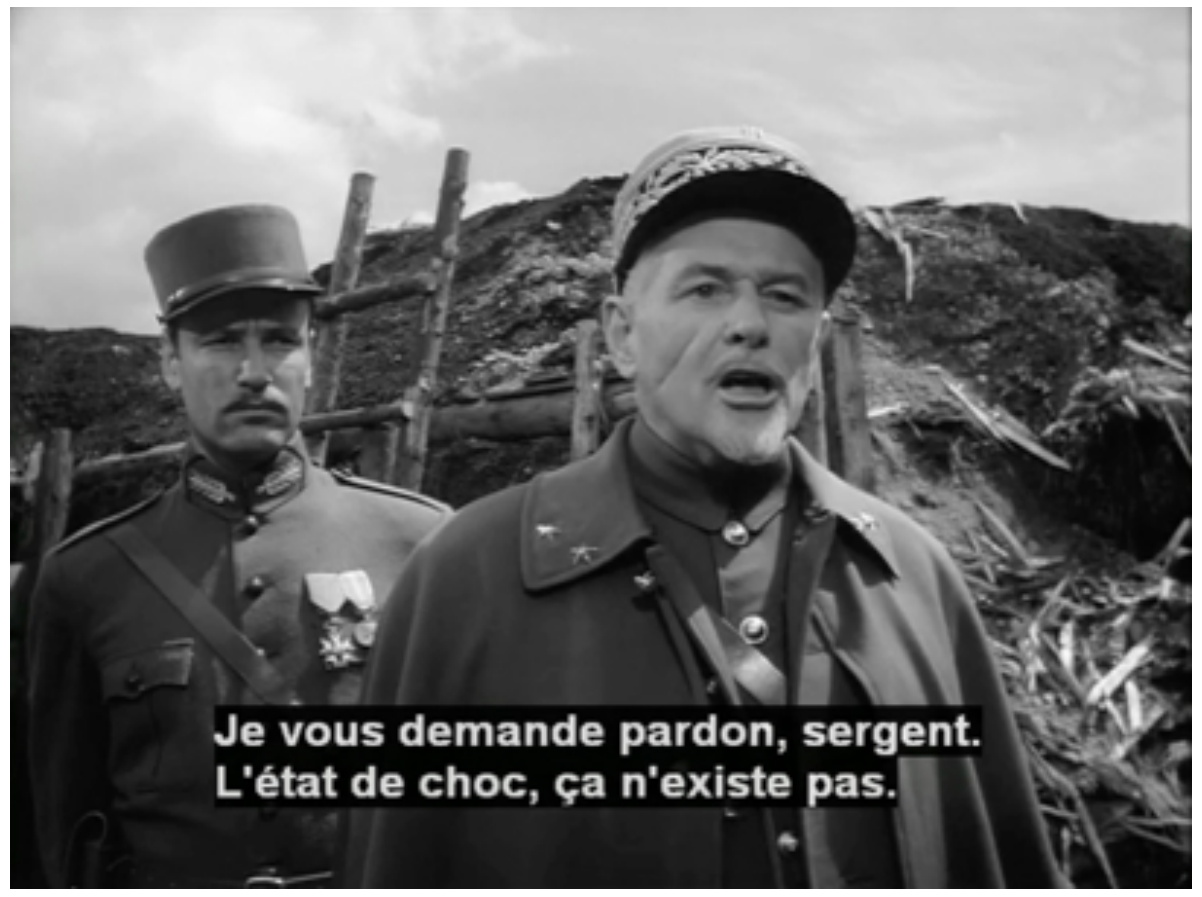

Document 4 : Général Mireau - "shell shock"

Paths of Glory (Stanley Kubrick, 1957, Bryna productions, United Artists), DR. 


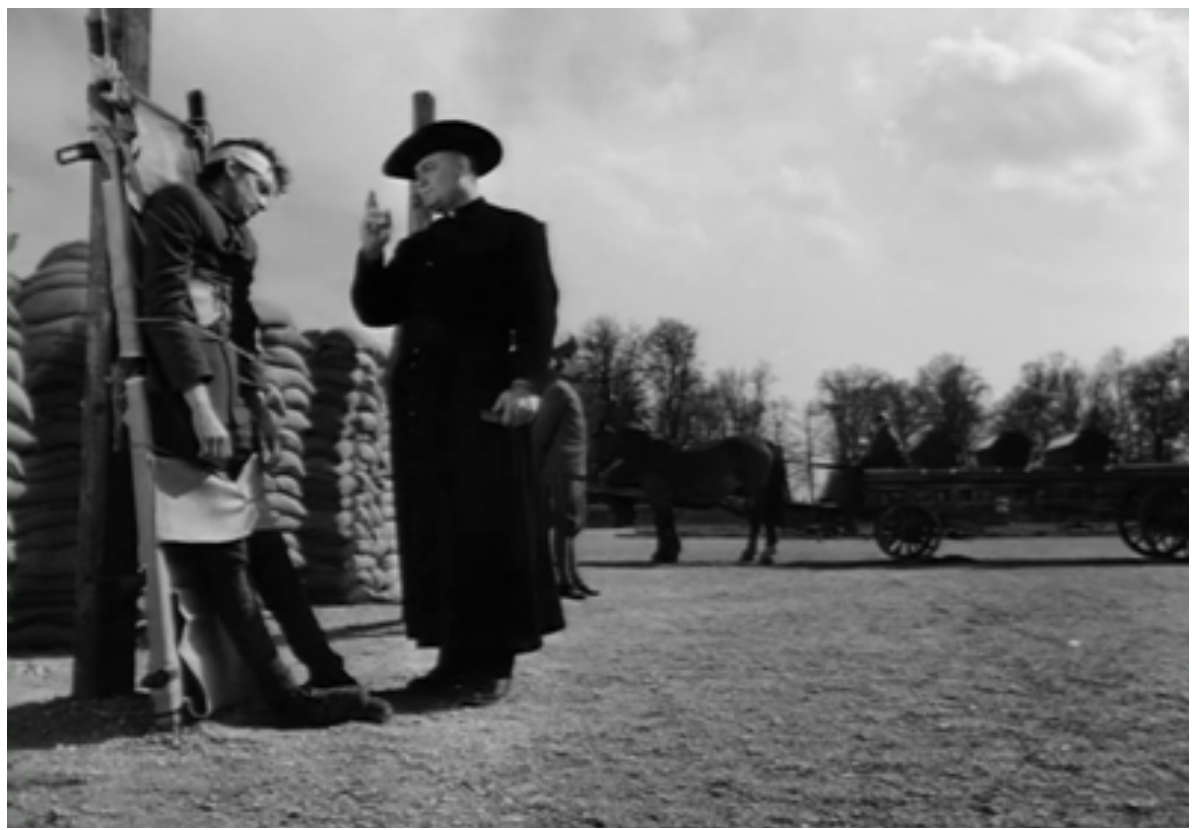

Document 5 : Évocation du sous-lieutenant Chapelant

Paths of Glory (Stanley Kubrick, 1957, Bryna productions, United Artists), DR.

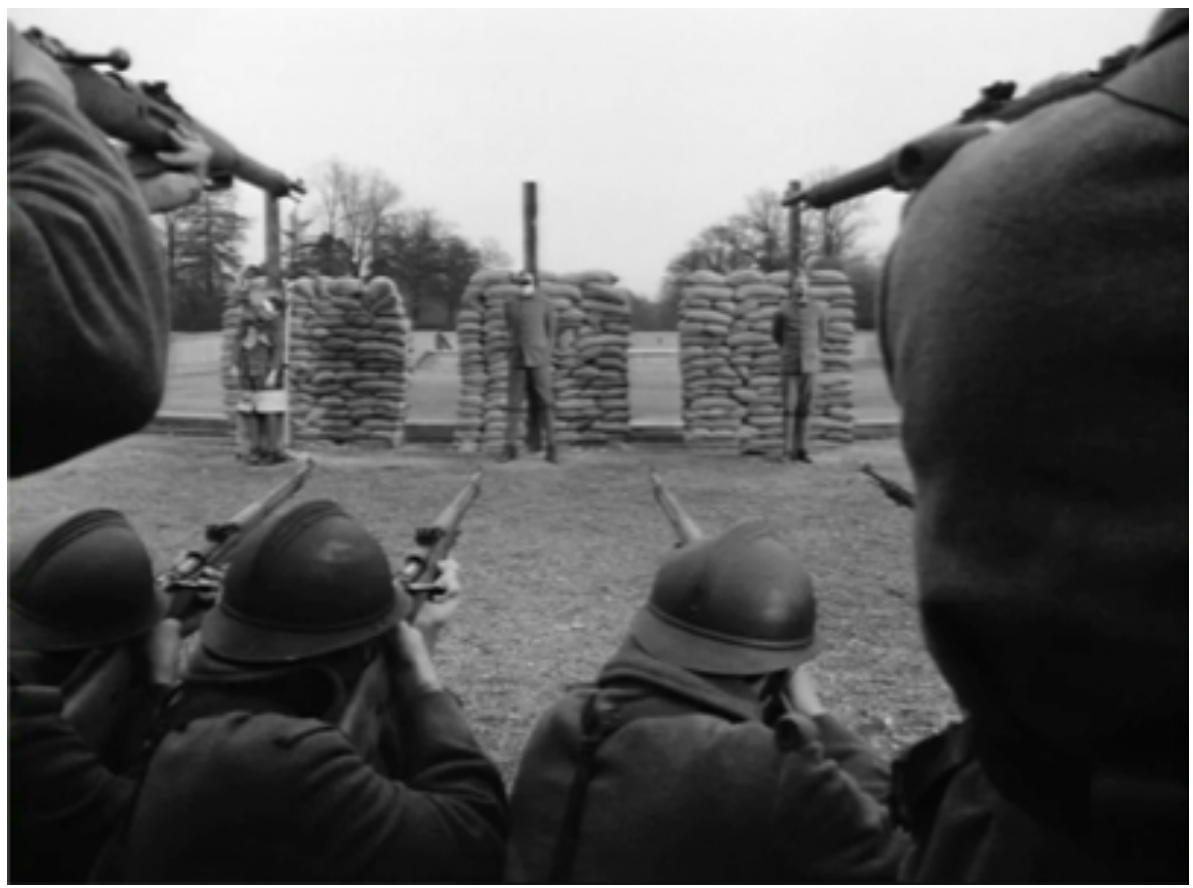

Document 6 : Exécution vue du peloton

Paths of Glory (Stanley Kubrick, 1957, Bryna productions, United Artists), DR. 


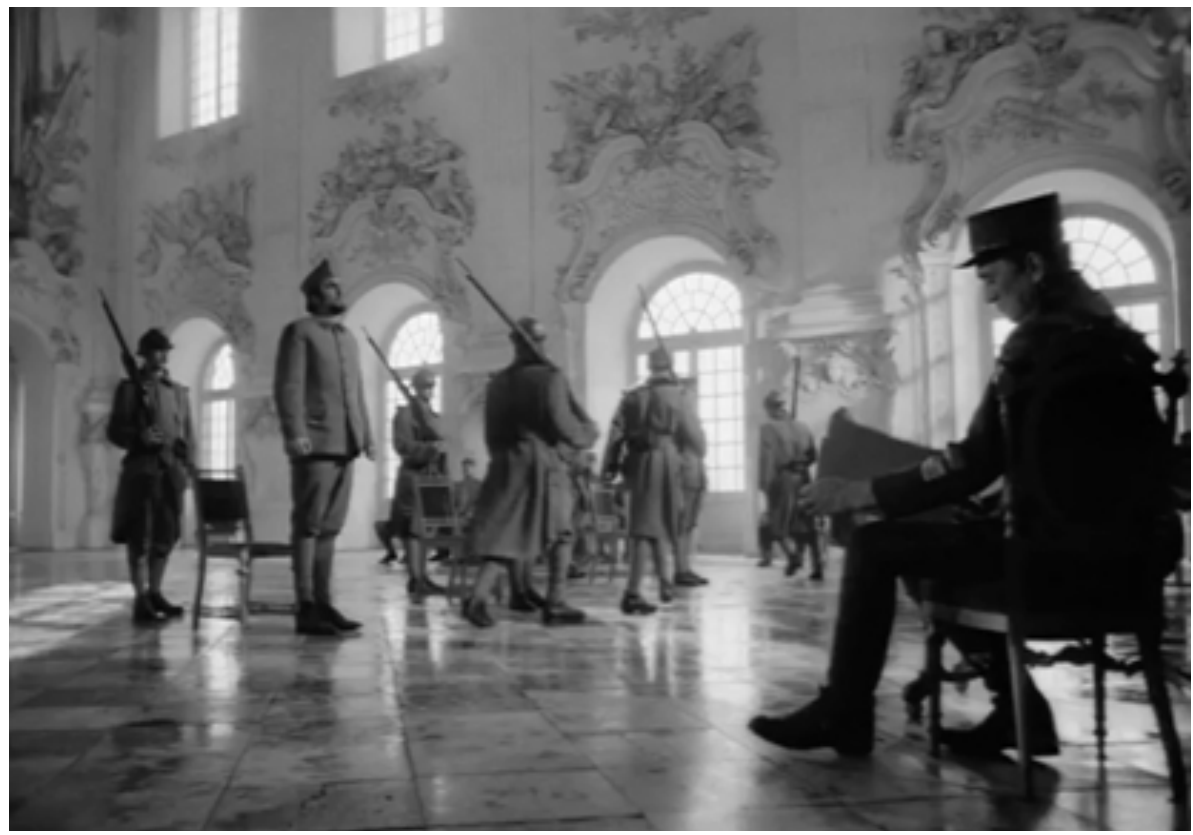

Document 7 : Tribunal-château

Paths of Glory (Stanley Kubrick, 1957, Bryna productions, United Artists), DR.

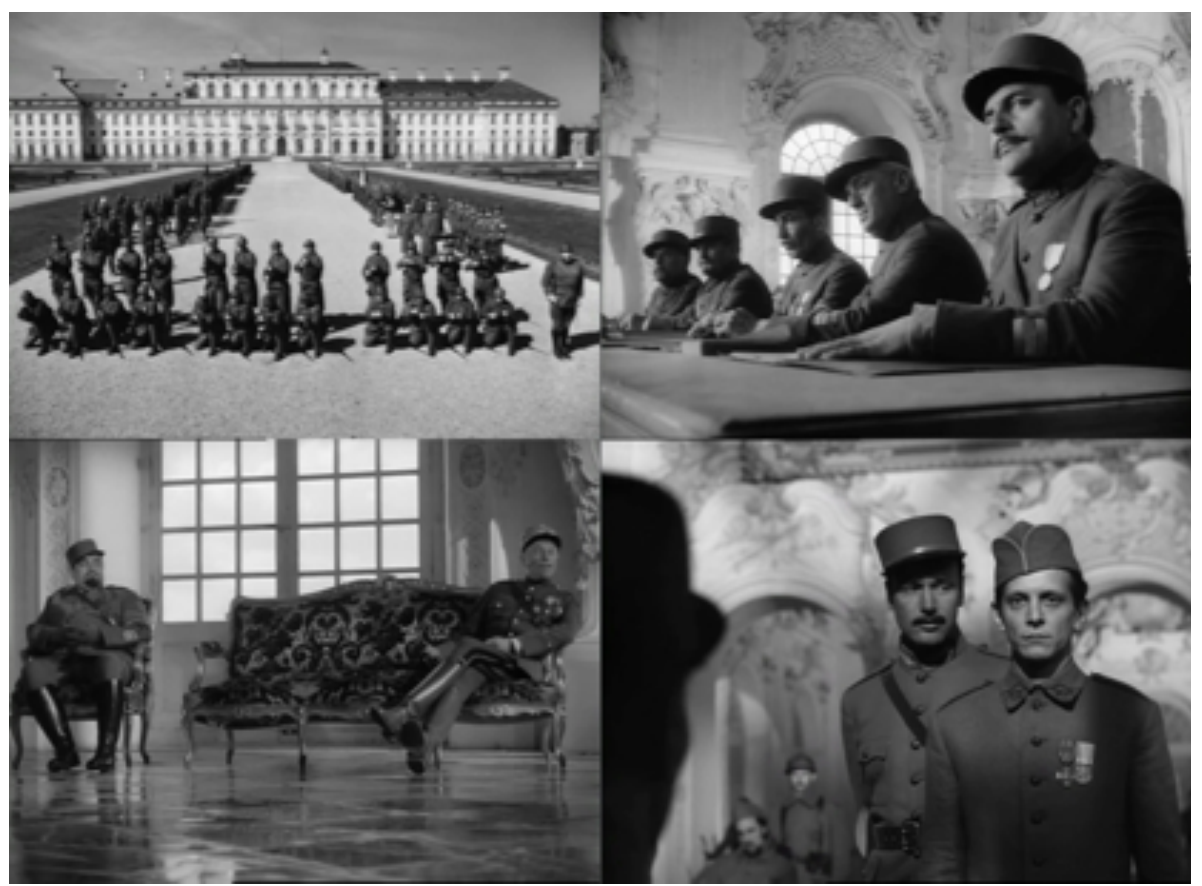

Document 8 : Du simulacre de procès à l'exécution Paths of Glory (Stanley Kubrick, 1957, Bryna productions, United Artists), DR. 